\title{
Effect of Probiotic Supplementation along with Calorie Restriction on Metabolic Endotoxemia, and Inflammation Markers in Coronary Artery Disease Patients: A Double Blind Placebo Controlled Randomized Clinical Trial
}

jalal moludi ( $\nabla$ jmoludi@yahoo.com )

Tabriz University of Medical Sciences Faculty of Medicine https://orcid.org/0000-0002-8333-414X

Hossein Samadi Kafil

Tabriz University of Medical Sciences

\section{Pourya Gholizadeh}

Tabriz Medical University: Tabriz University of Medical Sciences

Mohammad Alizadeh

Tabriz Medical University: Tabriz University of Medical Sciences

Hamed Jafari Vayghyan

Arak University of Medical Sciences

\section{Research}

Keywords: Coronary Artery Disease, lipopolysaccharide, gut microbiota, Metabolic endotoxmia, Probiotic

Posted Date: October 13th, 2020

DOI: https://doi.org/10.21203/rs.3.rs-71445/v2

License: (9) This work is licensed under a Creative Commons Attribution 4.0 International License.

Read Full License

Version of Record: A version of this preprint was published at Nutrition Journal on June 1st, 2021. See the published version at https://doi.org/10.1186/s12937-021-00703-7. 


\section{Abstract}

Purpose: Alterations in the gut microbiome (dysbiosis) has been associated with increased microbial translocation, leading to chronic inflammation in coronary artery disease (CAD). It has been proposed that modulation of gut microbiota by probiotic might modify metabolic endotoxemia. Therefore, the purpose of this study was to examine the effects of Lactobacillus rhamnosus GG (LGG) on metabolic endotoxemia, and marker of inflammation in CAD participants.

Methods: This study was a 12-weeks randomized, double-blind, and intervention on 44 patients with CAD. Patients were randomly allocated to receive either one LGG capsule $1.6 \times 10^{9}$ colony-forming unit (CFU) or the placebo capsules for 12 weeks. In addition, all the participants were also prescribed a calorierestricted diet. Serum levels of interleukin-1 $\beta$ (IL-1 $\beta$ ), Toll-like receptor 4 (TLR4), interleukin-10 (IL-10), and lipopolysaccharide (LPS), were assessed before and after the intervention.

Results: A significant decrease in IL1-Beta concentration (-1.88 \pm 2.25 , vs. $0.56 \pm 1.58 \mathrm{mmol} / \mathrm{L}, P=0.027)$, and LPS levels ( $-5.20 \pm 2.70$ vs. $2.96+5.27 \mathrm{mg} / \mathrm{L}, \mathrm{P}=0.016)$, was observed after the probiotic supplementation compared with the placebo. Participants who had $\geq 2.5 \mathrm{~kg}$ weight loss showed significantly improved cardiovascular-related factors, compared to patients with $<2.5 \mathrm{~kg}$ weight reduction, regardless of the supplement they took.

Conclusion: These data provide preliminary evidence that probiotic supplementation has beneficial effects on metabolic endotoxemia, and mega inflammation in participants with CAD.

\section{Introduction}

Cardiovascular disease (CVD) is the leading cause of death worldwide, a significant portion of which can be attributed to ischemic heart disease, often as a result of underlying coronary artery disease (CAD) due to atherosclerosis [1]. Despite the enormous growth in knowledge and advances in identifying traditional risk factors such as hypercholesterolemia, homocystinemia, hypertension, and hyperglycemia, which there are many unanswered questions about other cardiovascular risk factors yet $[2,3]$. Currently, the scholars have been paid attention to the role of gut microbiota alteration (dysbiosis) as one of the major etiological factors that are involved in the development of CAD [4].

Numerous data have supported the contribution of dysbiosis in the development of CAD by some mechanisms including increased gut permeability and metabolic endotoxemia $[5,6]$. This can may be explained by a microbiome-derived lipopolysaccharide (LPS), a major component of the external membrane in gram-negative bacteria. LPS can pass through the intestinal mucosa to enter the bloodstream, and may represent an important mediator of chronic inflammation [7, 8]. Chronic inflammation following ME might be a possible mechanism for the association between dysbiosis and CAD, which is triggered by dysbiosis [9]. LPS triggers toll-like receptors (TLRs) activation, and induces endothelial damage through increasing the expression of surface adhesion molecules such as cluster of differentiation 14 (CD14) on inflammatory cells, as well as, stimulates the release of proinflammatory 
cytokines [10]. Endotoxin may also induce plaque formation and progression of atherosclerotic lesions, and release of other molecules from endothelial cells involved in the proinflammatory process [11].

Probiotics are well-defined live microbe, which can administrated in adequate amounts and have positively effects on the health of the host by improving or restoring the gut flora [12]. In addition, probiotics exert anti-obesity and lipid-lowering effects, anti-inflammatory and anti-oxidative activity [13, 14]. In additions to probiotics help balance keeping of homeostasis in gut microbiota, they have been also considered as a possible treatment for CAD [15]. A small number of studies have explored the effects of probiotics on systemic levels of endotoxin in some clinical settings. Those that have observed the effect of probiotics administration on endotoxin levels and associated metabolic disorders have revealed conflicting results $[16,17]$. Probiotics keep preserve integration of gut barrier function and decrease intestinal permeability, which consequently decline endotoxin levels [18].

Weight loss diets and control of traditional risk factors are the treatment choices for CAD in overweight or obesity participants [19]. Several guiding principles are recommended following a weight loss program to achieve 5-10\% weight reduction [20]. Although the few animal studies appear to support the fact that dietary intervention leads to significant improvements in endotoxin levels [21]. In addition, the effect of dietary intervention on endotoxin levels has not been studied in human studies yet.

To the best of our knowledge, there have been little or no controlled randomized trials testing the effect of probiotic supplementation along with calorie restriction on metabolic endotoxemia in CAD participants. Therefore, the current study evaluated the effects of probiotic supplementation along with calorie restriction on metabolic endotoxemia, and inflammation markers in CAD participants .

\section{Materials And Methods}

\section{Participants}

We ran a double-blind randomized, placebo-controlled study to inspect the endotoxemia, and inflammation levels in 44 CAD participants. This trial was done at Shahid Madani hospital, as cardiology and heart surgery hospital of Tabriz University of Medical Sciences, Tabriz, Iran. Our study was in agreement with the Helsinki Declaration of the World Medical Association (2000) and was accepted by our local ethics committee of Tabriz University of Medical sciences (IR.TBZMED.REC.1397.184) and also was listed in the Iranian Registry of Clinical Trials (IRCT) (IRCT20121028011288N15). All patients admitted to this hospital with a diagnosis of CAD were considered for participation in the study and screened by a cardiologist for eligibility. Patients were selected a few days after the $\mathrm{PCl}$ and/or conditions were stable. The inclusion criteria were as follows: having CAD; maintaining them during the course of the trial; normal diet; and Body Mass Index (BMI) $=25-35 \mathrm{~kg} / \mathrm{m}^{2}$. Patients were excluded if they refused to participate or if they had a history of gastrointestinal disorders, if they had thyroid, renal, pancreatic, or liver diseases; if they were lactating or pregnant; if they are taking antibiotics, probiotics, prebiotics, and inflammatory drugs for one month before the intervention or during the intervention. A 
third party who was blind to the study gave the randomization sequence extracted from allocation software. The contributors, investigators and the medical providers were blinded after assignment to interventions. Informed consent was obtained from all subjects. All 44 contributors completed the study and none of them dropped their participation (Fig 1. CONSORT Flow diagram).

\section{Sample size}

To calculate sample size, mean (standard deviation; SD) of LPS was used from a previous clinical trial on the effect of prebiotic on endotoxmia of obese patients [22]. Calculated based on a confidence interval (Cl) of $95 \%$, and power of $80 \%$ in two-sided tests using Power analysis and sample size software (PASS; NCSS, LLC, US) version 15 , the sample size was 20 per group, which was increased to 22 , considering a probable $10 \%$ dropout rate

\section{Study design}

A blind trial at two levels (researcher, participants), placebo-controlled, randomized, clinical design was used to examine the effect of probiotic supplementation on endotoxemia in patients with CAD. Each qualified contributor was randomly allocated into probiotic or placebo group, according to 1:1 equal proportion rule. The order of random allocation was made by random sequence software. The random numbers were kept by a free person not complicated in the assessment of the patient, or in the data collection and analysis. In the current study, patients were randomly assigned into two groups to receive whichever probiotic supplements $(n=22)$ or placebo $(n=22)$ for 3 months. Both participants and researchers were unaware of the treatment allocation.

\section{Intervention}

All the participants received a moderate calorie restricted dietary plan during 12 weeks' intervention period. In this study, the program was designed to enable weight loss of $7-10 \%$ of weight, at a rate of $0.5-1 \mathrm{~kg} / \mathrm{wk}$ throughout the intervention. The weight-loss program was calculated to introduce a 500 - to $1000-k c a l$ energy deficit based on estimated energy requirements at the baseline of the intervention. Patients in the probiotic group received one probiotic capsule daily containing a Lactobacillus rhamnosus $1.6 \times 10^{9}$ colony-forming unit (CFU) with their lunch. In the placebo (control) group, the capsules contained maltodextrin (Tak Gen Zist Pharmaceutical Company, Tehran, Iran). The physical properties of the placebo were identical in terms of shape, color, size, packaging, and smell but contained no bacteria. Phone contacts were made to ensure adherence twice a month. Compliance to supplementation was established by requesting participants to return the medication containers. For ethical issues, the subjects were allowable to take their routine medications. However, taking any antioxidants and/or vitamin supplements were prohibited during the trial. The participants were allowed to discontinue the trial if they were unwilling to complete or experience any adverse effect during the intervention. Adverse effect evaluation was complete during the trial by questioning the participants and assessments for any adverse effect related to the intervention. At the beginning of the study, participants were recommended not to change their level of physical activity. 
In addition, a moderate calorie restriction diet plan intended for all the participants during 12 weeks' intervention period. The program was planned to facilitate weight loss of $7-10 \%$ of weight, at an amount of $0.5-1 \mathrm{~kg} / \mathrm{wk}$ throughout the intervention. Calorie intake was planned based on individual features of the participants and with the aim of daily energy restriction (500 kcal fewer than the total energy requirements [TEE] estimated by Mifflin-St Jeor equation). The diets were provided $55 \sim 60 \%$ of TEE from carbohydrate, $10 \sim 15 \%$ from protein, and $25 \sim 35 \%$ from fat.

\section{Dietary assessment}

Dietary intake was assessed using a dietary record at month 0,1 and 3 of the intervention. We used Nutritionist IV software adjusted for Iranian diets to acquire nutrient intakes of participants based on the average of three-day food diaries.

\section{Physical Activity Assessment}

The physical activity assessment was gotten to monitor patient's usual physical activity levels throughout the study. The validated short-form International Physical Activity Questionnaire (IPAQ) was used to measure the participant's physical activity. Based on previous studies, physical activities were classified as low, moderate, and high.

\section{Assessment of anthropometric indices}

Body weight was assessed via a scale with 250 gr accuracy (Seca, Hamburg, Germany) and patients were measured while wearing a minimum dress and without shoes. Height without shoes was measured by a tape with $0.5-\mathrm{cm}$ accuracy. BMI was computed by dividing weight $(\mathrm{Kg})$ by height ${ }^{2}(\mathrm{~m})$. To avoid measurement bias, all measurements were taken by a trained dietitian.

\section{Biochemical variables}

After an overnight fasting (12 hours), blood was collected and supplements was provided to the participants. Blood serum was obtained from whole blood through centrifugation at $2500 \mathrm{rpm}$ for $10 \mathrm{~min}$. FBS and lipid profile were examined on the day of sampling, and residual serum was stored at $-20 \mathrm{C}$ until the analyses were done. Endotoxin, and inflammatory markers were measured using enzyme-linked immunosorbent assay [17] kits as follows: Interleukin 1 beta (IL-1 $\beta$ ) (intra-assay variation $=5.8 \%$, normal range $=0.02-6 \mathrm{pg} / \mathrm{mL}$, inter-assay variation $=9.06 \%$, sensitivity $=0.01 \mathrm{pg} / \mathrm{mL}$ ), Toll-Like Receptor 4 (TLR4) (normal range $=0.05-15 \mathrm{ng} / \mathrm{mL}$, intra-assay variation $=4.58 \%$, inter-assay variation $=7.8 \%$, sensitivity $=0.027 \mathrm{ng} / \mathrm{mL}$ ), IL-10 (normal range $=0.2-100 \mathrm{ng} / \mathrm{dl}$, intra-assay variation $=10 \%$ ), and, LPS (inter-assay $\mathrm{CV}=10.0 \%$, detectable range $=12.00-1000 \mathrm{ng} / \mathrm{ml}$ ). 


\section{Statistical analysis}

The data were analyzed via SPSS software (version 21; SPSS Inc., Chicago, IL) and the outcomes were stated as mean \pm SD. To determine the normal distribution of variables, we used skewness and kurtosis test. Paired samples t-test was applied for within-group comparisons (end-point vs. baseline). Afterward adjusting for the confounders (weight, and calorie intakes) and baseline levels, we did analysis of covariance (ANCOVA) in which the confounding effect of these variables were taken into account which was used to determine the statistically significant pairwise differences. The analyses were conducted using an intention-to-treat approach [23]. Circulating endotoxin level was intended as a primary outcome, while inflammatory markers were defined as secondary outcomes. For all statistical tests, a $P$ value less than 0.05 was interpreted as statistically significant.

\section{Results}

Based on the ITT principle, we included all participants $(n=44)$ in analyzes.. Figure 1 are shown the study flowchart. Adherence to dosage in our study was over $80 \%$ of pills in both groups (Table. 1)

Baseline medical and demographic data are presented in Table.1. There were no significant differences between the groups regarding weight, BMI, and family history of CAD. At starting point, there was no significant difference between the two groups in term of the level of physical activity. Similarly, physical activity level of the participants who finished the trial stayed unchanged all over the study $(P>0.050$ for both groups) (data not shown).

Data on dietary intakes (macronutrient distribution, as percentage of calories, changes and dietary fiber) of the patients are summarized in Table 2. Intake of calories and macronutrients were significantly decreased in both groups. We found significant changes in the mean of energy intake of the groups during the intervention (decreasing of -284.40 and $-384.71 \mathrm{Kcal}$ in probiotics and placebo groups, respectively, $P=0.225$ ). Greater decrease of dietary intake was observed in the prebiotic group in comparison to placebo group $(\mathrm{P}>0.05)$.

Table 3 provides a summary of pre- and post-intervention metabolic endotoxemia and inflammation markers in both groups. Neither the between-group differences nor the within-group variations reached statistical significance for TLR4. A significant decrease in IL1-Beta concentration $(-1.88 \pm 2.25$, vs. $0.56 \pm$ $1.58 \mathrm{mmol} / \mathrm{L}, \mathrm{P}=0.027)$, and LPS levels $(-5.20 \pm 2.70 \mathrm{vs}$. $2.96+5.27 \mathrm{mg} / \mathrm{L}, \mathrm{P}=0.016)$ were detected following the probiotic supplementation in comparison to placebo group, respectively. Probiotics administration was resulted in a significant decrease in biomarker levels of inflammation, and metabolic endotoxemia in comparison to placebo group.

Results stratified by weight reduction of at minimum $2.5 \mathrm{~kg}$ was presented in Table 4 . Overall, $28 \%$ of the participants, who finished the trial had at least $2.5 \mathrm{~kg}$ weight loss (8 patients in the probiotic group and 4 
patients in the placebo group). Nevertheless, besides of what supplement they receive, participants who reached weight loss had great decrease in some biomarkers in comparison to whom lost $<2.5 \mathrm{~kg}$.

\section{Discussion}

As far as we know, this is the first study to provide insight into the anti-inflammatory and antiendotoxemia effects of probiotic in CAD patients. The results of the current study indicated, for the first time, that weight loss diet along with probiotic supplementation lead to improved endotoxemia, as described by reduction of the LPS and TLR4 levels. Probiotic supplementation also altered the gut microbiota profile. In addition, inflammatory markers were influenced favorably by probiotic supplements, among patients with CAD under a calorie-restricted diet. We also found that those who were obedient with the weight loss diet and lost a minimum of $2.5 \mathrm{~kg}$ by the end of the intervention had significantly improved metabolic profile in comparison to those with slight compliance with the diet.

In the current study, we ran the weight loss program plus probiotic supplementation. Although anthropometric and body composition indices reduction in the probiotic group was more advantageous than the placebo group, bur this issue did not range to statically significant levels. Similar consequences have been stated with probiotic supplementation in former studies [24]. In the current study, we were statistically observed major changes in nutrient intakes for the period of the intervention and there was a downward trend in the total energy intake in all participants due to the running of weight loss diet for both groups. Though weight loss was similar between the two groups, our results suggest that weight loss diet offers a favorable effect on metabolic disorders, but add-on the diet plus probiotics may promote cardiovascular risk factors (Table 4).

The other result of probiotic supplementation in CAD patients was an improvement in inflammatory markers. To the extent of our knowledge, no study has assessed the effect of probiotics supplementation on endotoxemia in patients with CAD. Former studies have stated microbial dysbiosis in CAD participants [7]. Probiotic supplementation by modulating gut microbiota enhances the immune system function and decreases inflammation and endotoxmia $[9,16]$. In our study, we established a statistically significant reduction in LPS levels in the probiotic group in comparison to placebo, but not in TLR4, which suggested probable anti-inflammatory and anti-endotoxemia properties of probiotics. Activation by LPS was resulted in an increase in plasma levels of cytokines like interleukin (IL-) 1 Beta, which was observed in CAD patients [7]. The outcomes of our study show that probiotic supplementation could led to decreased IL-1 Beta and hs-CRP levels. The anti-inflammatory effects of probiotics may involve the production of short chain fatty acids (SCFA) in the gut microbiota and the decreased expression of inflammatory cytokines [25]. This finding is consistent with the outcomes reported by Zarrati et al [26], who established that Lactobacillus acidophilus administration as a probiotic for 2 months resulted in a significant decrease in inflammatory markers among obese participants . In addition, a significant decrease in hsCRP levels was detected after the intake of probiotic yogurt for 9 weeks among pregnant women [27]. 
Others have failed to find a significant effect of two-month probiotic supplementation on CRP levels in polycystic ovary syndrome (PCOS) participants [28]. The different results might be clarified by diverse dosages of probiotics supplementations and different clinical setting of individuals, who took part in those studies. Microbial translocation has been proposed to be a driver of inflammation and immune activation in CAD patients $[5,29]$.

Whereas the contribution of pro-inflammatory cytokines to progression of CAD is well- recognized [30], little is identified about the effect of microbial translocation (increases LPS) on the higher cytokine secretion [31]. To the best of our knowledge, no clinical trial had appraised the effects of probiotics on TLR-4 in meta-inflammation. TLR4 has been suggested to act as a molecular link among LPS, inflammation and the innate immune system [11]. It seems that probiotic supplements reduce inflammation possible via competitive mechanisms, which prevents binding LPS to theTLR4/CD14 complex [9, 32]. In addition, previous studies have indicated that calorie restriction can downregulate LPSproducing enzymes of the commensal bacteria, leading to decreased endotoxemia and inflammation [33]. There is one single study which indicates that being on a very-low calorie diet ( $800 \mathrm{Cal} / \mathrm{day})$ for one month decreases LPS binding protein and zonulin in obese women [34]. Apart from that, there are few clinical trials available about the effects of calorie restriction (25-30\% below TEE) on serum LPS. Therefore, we could not compare our results for LPS, to the previous studies about the effect of calorie restrictions on these factors.

As mentioned above, dysbiosis leads to endotoxemia and chronic inflammation. These two factors have been proposed to make participants vulnerable to CADs. Probiotics might reverse these effects by helping to enhance or restore health gut microbiota composition. Cui et al.[35], revealed that probiotic supplementation with Bifidobacterium spp in IBD patients for 2 months, meaningfully increased the stool concentration of Bifidobacterium spp and Lactobacillus spp, compared to placebo. However, in the study of Kato et al.[36], consumption of Bifidobacterium-fermented milk for 3 months did not alter the level of stool Bacteriodes.

Our study has some of the limitations, including not assessing the gut microbiota, as well as the duration and doses of the supplementations. Furthermore, the sample size is small, which may interfere with generalizability. So, given the gradually common use of Firmicutes/Bacteriodes ratio, the analysis of these bacterial contents may well be used in the future as indicators of a dysbiotic microbiome that contributes to low grade inflammation and CAD progression. Apart from that, this supplementation is worthy of additional survey.

\section{Conclusion}

The present trial revealed that 12 weeks' probiotic supplementation (L. rhamnose) has a positive effect on endotoxemia and chronic inflammation in CAD patients. In addition, our results propose that calorie restriction plus probiotic supplementations might lead to improved metabolic endotoxemia and better 
than probiotic supplementation alone. Further studies are needed to replicate the present findings and investigate the mechanisms of the observed effects and test the generalizability of the current findings in CAD patients and patients with other types of cardiovascular disease.

\section{Abbreviations}

MI

Myocardial infarction; LVEF:Left ventricle Ejection fraction; ELISA:enzyme-linked immunosorbent assay, HF:heart failure, BMI:Body Mass Index BP:Blood Pressure DBP:Diastolic Blood Pressure, FBS:Fasting Blood Sugar, HDL:High-Density Lipoprotein, LDL:Low-Density Lipoprotein, SBP:Systolic Blood Pressure, TC:Total Cholesterol, TG:Triglyceride

\section{Declarations}

Ethics declarations

Conflict of Interest

The authors declare that there is no conflict of interest.

\section{Ethical Approval}

Our study was in agreement with the Helsinki Declaration of the World Medical Association (2000) and was accepted by our local ethics committee of Tabriz University of Medical sciences as a proposal for PhD grade in Nutritional Sciences (IR.TBZMED.REC.1397.184) and also was listed in the Iranian Registry of Clinical Trials (IRCT) (IRCT20121028011288N15).

\section{Consent for publication}

All authors support the submission to this journal.

\section{Informed Consent}

Informed consent was obtained from all individual participants included in the study using opt-out procedure.

\section{Funding/Support}

This research was partially supported by Tabriz University of Medical sciences ((grant number 184).

\section{Acknowledgements}

The authors would like to thank the Clinical Research Development Center of Imam Reza Hospital, Kermanshah University of Medical Sciences, Kermanshah, Iran for their wise pieces of advice. 


\section{Authorcontribution}

Jalal moludi designed the study. Hossein Samadi Kafil and Pourya Gholizadeh completed the entire clinical studies. Jalal moludi collected and analyzed the data. Mohammad Alizadeh prepared the manuscript. Jalal moludi conducted statistical analysis. All of authors edited the manuscript.

\section{Availability of data and materials}

All data generated and analyzed during this study are included in the manuscript.

\section{References}

1. Lopez AD, et al. Global and regional burden of disease and risk factors, 2001: systematic analysis of population health data. The Lancet. 2006;367(9524):1747-57.

2. Duntas LH, Wartofsky L. Cardiovascular risk and subclinical hypothyroidism: focus on lipids and new emerging risk factors. What is the evidence? Thyroid. 2007;17(11):1075-84.

3. Long A, Long B, Koyfman A. Non-traditional risk factors for atherosclerotic disease: A review for emergency physicians. Am J Emerg Med. 2018;36(3):494-7.

4. Lau K, et al. Bridging the gap between gut microbial dysbiosis and cardiovascular diseases. Nutrients. 2017;9(8):859.

5. Serino $M$, et al. Far from the eyes, close to the heart: dysbiosis of gut microbiota and cardiovascular consequences. Curr Cardiol Rep. 2014;16(11):540.

6. Moludi J, et al. New insights on atherosclerosis: A cross-talk between endocannabinoid systems with gut microbiota. Journal of cardiovascular thoracic research. 2018;10(3):129.

7. Jayashree B, et al. Increased circulatory levels of lipopolysaccharide (LPS) and zonulin signify novel biomarkers of proinflammation in patients with type 2 diabetes. Molecular cellular biochemistry. 2014;388(1-2):203-10.

8. $\mathrm{Ng} \mathrm{QX}$, et al. The role of inflammation in irritable bowel syndrome (IBS). Journal of inflammation research. 2018;11:345.

9. Tang WW, Kitai T, Hazen SL. Gut microbiota in cardiovascular health and disease. Circulation research. 2017;120(7):1183-96.

10. Tsukamoto H, et al. Lipopolysaccharide (LPS)-binding protein stimulates CD14-dependent Toll-like receptor 4 internalization and LPS-induced TBK1-IKKe-IRF3 axis activation. J Biol Chem. 2018;293(26):10186-201.

11. Pasterkamp G, Van Keulen J, De Kleijn D. Role of Toll-like receptor 4 in the initiation and progression of atherosclerotic disease. Eur J Clin Invest. 2004;34(5):328-34.

12. Sanders ME, et al. Probiotics and prebiotics in intestinal health and disease: from biology to the clinic. Nature reviews Gastroenterology hepatology. 2019;16(10):605-16.

13. Shokryazdan P, et al. Probiotics: from isolation to application. J Am Coll Nutr. 2017;36(8):666-76. 
14. Prado MRM, Boller C, Anti-inflammatory effects of probiotics, in Discovery and Development of AntiInflammatory Agents from Natural Products. 2019, Elsevier. p. 259-282.

15. Reid G. Probiotics: definition, scope and mechanisms of action. Best Pract Res Clin Gastroenterol. 2016;30(1):17-25.

16. Lata J, et al. The effect of probiotics on gut flora, level of endotoxin and Child-Pugh score in cirrhotic patients: results of a double-blind randomized study. Eur J Gastroenterol Hepatol. 2007;19(12):1111-3.

17. Villar-García J, et al. Effect of probiotics (Saccharomyces boulardii) on microbial translocation and inflammation in HIV-treated patients: a double-blind, randomized, placebo-controlled trial. JAIDS Journal of Acquired Immune Deficiency Syndromes. 2015;68(3):256-63.

18. Durack J, Lynch SV. Promotion of Epithelial Barrier Integrity Via Probiotic-derived Products. J Pediatr Gastroenterol Nutr. 2017;64(3):335-6.

19. Barnason S, et al. Weight Management Telehealth Intervention for Overweight and Obese Rural Cardiac Rehabilitation Participants: A Randomized Trial. Journal of clinical nursing. 2019;28(910):1808.

20. Noël PH, Pugh JA. Management of overweight and obese adults. Bmj. 2002;325(7367):757-61.

21. Cani PD, et al. Changes in gut microbiota control metabolic endotoxemia-induced inflammation in high-fat diet-induced obesity and diabetes in mice. Diabetes. 2008;57(6):1470-81.

22. Szulińska M, et al. Dose-dependent effects of multispecies probiotic supplementation on the lipopolysaccharide (LPS) level and cardiometabolic profile in obese postmenopausal women: A 12week randomized clinical trial. Nutrients. 2018;10(6):773.

23. Wright C, Sim J. Intention-to-treat approach to data from randomized controlled trials: a sensitivity analysis. J Clin Epidemiol. 2003;56(9):833-42.

24. Park S, Bae J-H. Probiotics for weight loss: a systematic review and meta-analysis. Nutrition research. 2015;35(7):566-75.

25. Rooks MG, Garrett WS. Gut microbiota, metabolites and host immunity. Nat Rev Immunol. 2016;16(6):341.

26. Zarrati M, et al. Lactobacillus acidophilus La5, Bifidobacterium BB12, and Lactobacillus casei DN001 modulate gene expression of subset specific transcription factors and cytokines in peripheral blood mononuclear cells of obese and overweight people. Biofactors. 2013;39(6):633-43.

27. Asemi Z, et al. Effect of multispecies probiotic supplements on metabolic profiles, hs-CRP, and oxidative stress in patients with type 2 diabetes. Annals of nutrition metabolism. 2013;63(1-2):1-9.

28. Ghanei $\mathrm{N}$, et al. The probiotic supplementation reduced inflammation in polycystic ovary syndrome: a randomized, double-blind, placebo-controlled trial. J Funct Foods. 2018;42:306-11.

29. Yuzefpolskaya M, et al., Gut microbiota, endotoxemia, inflammation, and oxidative stress in patients with heart failure, left ventricular assist device, and transplant. The Journal of Heart and Lung Transplantation, 2020. 
30. Kanda T, Takahashi T. Interleukin-6 and cardiovascular diseases. Japanese heart journal. 2004;45(2):183-93.

31. Turrin NP, et al. Pro-inflammatory and anti-inflammatory cytokine mRNA induction in the periphery and brain following intraperitoneal administration of bacterial lipopolysaccharide. Brain research bulletin. 2001;54(4):443-53.

32. Ohland CL, MacNaughton WK. Probiotic bacteria and intestinal epithelial barrier function. American Journal of Physiology-Gastrointestinal Liver Physiology. 2010;298(6):G807-19.

33. Bleau $\mathrm{C}$, et al. Crosstalk between intestinal microbiota, adipose tissue and skeletal muscle as an early event in systemic low-grade inflammation and the development of obesity and diabetes. Diab/Metab Res Rev. 2015;31(6):545-61.

34. Ott B, et al. Effect of caloric restriction on gut permeability, inflammation markers, and fecal microbiota in obese women. Scientific reports. 2017;7(1):11955.

35. Cui $\mathrm{H}-\mathrm{H}$, et al. Effects of probiotic on intestinal mucosa of patients with ulcerative colitis. World $\mathrm{J}$ Gastroenterol. 2004;10(10):1521.

36. Kato K, et al., Randomized placebo-controlled trial assessing the effect of bifidobacteria-fermented milk on active ulcerative colitis. Alimentary pharmacology \& therapeutics, 2004. 20(10): p. 11331141.

\section{Tables}


Table 1- General characteristics of the study subjects

\begin{tabular}{|c|c|c|c|}
\hline P-value & $\begin{array}{l}\text { Placebo group } \\
(n=22)\end{array}$ & $\begin{array}{l}\text { Probiotic group } \\
(n=22)\end{array}$ & Variable \\
\hline $0.876^{d}$ & $57.10 \pm 7.80$ & $56.70 \pm 9.10$ & Age $(\text { years })^{a}$ \\
\hline $0.390^{d}$ & $79.20 \pm 12.10$ & $75.60 \pm 12.30$ & Weight $(\mathrm{kg})^{\mathrm{b}}$ at study baseline \\
\hline 0.156 & $77.54 \pm 11.20$ & $72.35 \pm 12.40$ & Weight $(\mathrm{kg})^{\mathrm{b}}$ after intervention \\
\hline $0.431^{c}$ & $4(18)$ & $5(22)$ & Smoking \\
\hline 0.830 & $760.6(201.21)$ & 773.7 (198.3) & Physical activity (METs) \\
\hline 0.527 & $38.31(6.7)$ & $37.0(7.1)$ & Ejection Fraction (\%) \\
\hline $0.431^{c}$ & $18(82)$ & $17(78)$ & Non-Smoking n(\%) \\
\hline \multirow[t]{4}{*}{$0.491^{c}$} & & & PAL n(\%) \\
\hline & $2(10)$ & $7(31)$ & Low \\
\hline & $19(86)$ & $13(59)$ & Moderate \\
\hline & $1(4)$ & $2(10)$ & High \\
\hline
\end{tabular}

CAD: Coronary artery diseases, METs: Metabolic equivalents (MET-minutes/week); MD:

Mean/Median of difference

a- Values are expressed as mean (SD)

b- Values are expressed as frequency (\%)

c- Chi-square test

d- Independent samples t-test 
Table 2. Changes in calorie, percent from macronutrients, and dietary fiber throughout the study

$\begin{array}{llll}\text { Variable } & \text { Probiotic group }(n=22) & \text { Placebo group }(n=22) & \text { P-value } \\ \begin{array}{l}\text { Energy } \\ \text { (Kcal/d) }\end{array} & 2214.14(428.6) & 2060.7(454.38) & 153.4(-116.1 \text { to } 422.8) \text {, } \\ \text { Baseline } & 1829.34(174.6) & 1776.1(184.3) & 0.257^{\star \star} \\ \text { End } & & & 53.27(-32.1 \text { to } 138.68), \\ & & 0.225^{\star \star \star}\end{array}$

\section{Carbohydrates}

(\%)

$53.33(13.43)$

Baseline $\quad 54.72$ (10.22)

54.1 (11.51)

$53.88(11.34)$

End

$\mathrm{MD}(95 \% \mathrm{Cl})$
$P^{\star}$
Protein (\%)

Baseline

End

13.55 (3.9)

15.08 (3.68)

$1.39(-7.12,7.66), 0.667$

$-0.25(-6.34,5.12), 0.672$
$0.80(-7.25,5.78)$,

$0.987^{\star \star}$

$-0.92(-7.31,7.36)$, $0.807^{\star \star \star}$

$153.4(-116.1$ to 422.8$)$,

$53.27(-32.1$ to 138.68$)$,

$0.807+2$


$\operatorname{MD}(95 \% \mathrm{Cl}), \quad 3.37(-0.06,0.34), 0.090 \quad 2.00(-0.06,0.41), 0.147$

$P^{*}$

Mean (SD) and Mean difference $(95 \% \mathrm{Cl})$ are presented for data.

${ }^{*} \mathrm{P}$ based on Paired samples t-test

${ }^{* *} \mathrm{P}$ based on Independent samples t-test

${ }^{* * *} \mathrm{P}$ based on ANCOVA adjusted for baseline values 


\begin{tabular}{|c|c|c|c|}
\hline Variable & Probiotic group $(n=22)$ & Placebo group $(n=22)$ & P-value \\
\hline \multicolumn{4}{|l|}{ IL-10 (ng/dl) } \\
\hline Baseline & $5.11 \pm 4.60$ & $4.76 \pm 3.60$ & $0.11(-2.4$ to 2.7$), 0.930^{\star \star}$ \\
\hline End & $5.25 \pm 2.91$ & $5.78 \pm 2.29$ & $0.56(-2.6$ to 1.6$), 0.605^{\star \star \star}$ \\
\hline $\mathrm{MD}(95 \% \mathrm{Cl}), P^{*}$ & $0.60(-1.4,2.6) 0.542$ & $\begin{array}{l}1.04(-1.31,3.59) \\
0.399\end{array}$ & \\
\hline \multicolumn{4}{|l|}{$\begin{array}{l}\text { IL1-Beta } \\
(\mathrm{pg} / \mathrm{mL})\end{array}$} \\
\hline Baseline & $5.62 \pm 3.72$ & $5.31 \pm 2.09$ & $-0.31(-1.4$ to 2.6$), 0.723^{\star \star}$ \\
\hline End & $3.75 \pm 2.10$ & $5.81 \pm 3.60$ & $\begin{array}{l}2.05(-3.8 \text { to }-0.25) \\
\mathbf{0 . 0 2 7}\end{array}$ \\
\hline $\mathrm{MD}(95 \% \mathrm{Cl}), P^{*}$ & $-1.88(-3.25,-0.48) 0.010$ & $\begin{array}{l}0.56(-1.58,2.56) \\
0.546\end{array}$ & \\
\hline \multicolumn{4}{|l|}{ LPS (ng/ml) } \\
\hline Baseline & $21.92(11.64)$ & $26.18(16.85)$ & $4.25(-13.1,4.4), 0.335^{\star \star}$ \\
\hline End & $16.04(6.8)$ & $23.22(12.22)$ & $7.27(-13.4,-1.4), 0.016^{\star \star \star}$ \\
\hline $\mathrm{MD}(95 \% \mathrm{Cl}), P^{*}$ & $\begin{array}{l}-5.92(-10.74,-1.09), \\
0.019\end{array}$ & $-2.96(-11.6,5.7), 0.165$ & \\
\hline \multicolumn{4}{|l|}{ TLR4 (ng/ml) } \\
\hline Baseline & $11.59(7.22)$ & $10.58(8.30)$ & $-0.70(-2.4,5.6), 0.765^{\star \star}$ \\
\hline End & $9.8(7.24)$ & $11.85(5.6)$ & $2.2(-5.3,1.8), 0.301^{\star \star \star}$ \\
\hline $\mathrm{MD}(95 \% \mathrm{Cl}), P^{*}$ & $-1.72(-6.21,3.10), 0.488$ & $1.27(-1.7,4.7), 0.581$ & \\
\hline \multicolumn{4}{|c|}{ LPS: Lipopolysaccharide, Mean (SD) and Mean difference (95\% Cl) are presented for data. } \\
\hline \multicolumn{4}{|c|}{ * $\mathrm{P}$ based on Paired samples t-test } \\
\hline \multicolumn{4}{|c|}{${ }^{\star \star} \mathrm{P}$ based on Independent samples t-test } \\
\hline \multicolumn{4}{|c|}{${ }^{\star \star \star} \mathrm{P}$ based on ANCOVA adjusted for baseline values } \\
\hline
\end{tabular}




\section{Table 4- Changes in biomarkers and variables among the patients who completed the study, stratified by a decrease of $2.5 \mathrm{~kg}$ in body weight}

\begin{tabular}{|c|c|c|c|}
\hline MD (95\% Cl), P-value ${ }^{a}$ & $\begin{array}{l}\text { weight loss } \leq 2.5 \\
(n=32)\end{array}$ & $\begin{array}{l}\text { weight loss } \geq 2.5 \\
(n=12)\end{array}$ & Variable \\
\hline$-14.33(-1.1$ to -27.2$), 0.032$ & $111.52 \pm 15.16$ & $97.28 \pm 20.10$ & FBS $(\mathrm{mg} / \mathrm{dl})$ \\
\hline$-18.23(-13.14$ to 50.21$), 0.241$ & $159.66 \pm 47.11$ & $140.83 \pm 43.16$ & Total cholesterol (mg/dl) \\
\hline-25.52 (-11.1 to 62.33$), 0.174$ & $160.83 \pm 59.25$ & $134.34 \pm 37.16$ & $\mathrm{TG}(\mathrm{mg} / \mathrm{dl})$ \\
\hline-15.11 (-15.1 to 45.2$), 0.326$ & $87.44 \pm 44.39$ & $72.36 \pm 44.16$ & LDL (mg/dl) \\
\hline$-2.31(-2.22$ to 7.10$), 0.353$ & $44.31 \pm 7.59$ & $42.00 \pm 6.27$ & $\mathrm{HDL}(\mathrm{mg} / \mathrm{dl})$ \\
\hline$-9.13(-0.26$ to 18.31$), 0.056$ & $122.47 \pm 12.16$ & $113.33 \pm 13.18$ & $\mathrm{SBP}(\mathrm{mmHg})$ \\
\hline-0.99 (-8.66 to 6.51$), 0.794$ & $77.10 \pm 8.10$ & $78.83 \pm 7.16$ & $\mathrm{DBP}(\mathrm{mmHg})$ \\
\hline-3.21 (1.47to 6.2), 0.035 & $16.71 \pm 4.33$ & $13.50 \pm 3.80$ & BDI \\
\hline$-2.46(-7.7$ to 2.31$), 0.301$ & $39.28 \pm 7.23$ & $41.75 \pm 6.13$ & EF (口) \\
\hline-0.65 (0.16 to 0.24$), 0.011$ & $1.79 \pm 1.25$ & $1.14 \pm 0.45$ & hs-CRP (mg/dl) \\
\hline$-11.83(-35.1$ to 59.71$), 0.616$ & $137.00 \pm 72.39$ & $125.16 \pm 44.16$ & MDA (mg/dl) \\
\hline$-25.83(-83.1$ to 31.32$), 0.367$ & $193.81 \pm 89.50$ & $219.66 \pm 64.83$ & $\mathrm{TAC}(\mathrm{mg} / \mathrm{dl})$ \\
\hline$-2.91(-16.1$ to 22.2$), 0.769$ & $26.81 \pm 33.29$ & $23.90 \pm 10.18$ & TMAO (mmHg) \\
\hline$-0.84(-1.21$ to 2.94$), 0.429$ & $5.10 \pm 3.43$ & $4.17 \pm 1.93$ & IL1-Beta (mmHg) \\
\hline$-2.04(-0.44$ to 4.50$), 0.101$ & 6. $09 \pm 3.99$ & $4.05 \pm 2.11$ & IL-10 (mmHg) \\
\hline$-5.74(-0.22$ to 11.54$), 0.059$ & $16.25 \pm 9.75$ & $10.47 \pm 4.30$ & MMP (mmHg) \\
\hline$-0.77(-6.1$ to 8.81$), 0.833$ & $20.18 \pm 10.42$ & $19.50 \pm 11.50$ & TGF-Beta \\
\hline-0.32 (-1.09 to 0.44$), 0.404$ & $4.50 \pm 1.23$ & $4.17 \pm 1.07$ & Procolagen \\
\hline-0.27 (-6.7 to 7.2$), 0.938$ & $19.68 \pm 9.75$ & $19.41 \pm 11.42$ & LPS (mg/dl) \\
\hline$-1.00(-3.1$ to 5.40$), 0.650$ & $11.11 \pm 6.39$ & $10.11 \pm 6.81$ & TLR4 (mg/dl) \\
\hline$-71.46(-161.1$ to 18.2$), 0.116$ & $110.17 \pm 123.50$ & $181.66 \pm 151.83$ & L. Rhamnosus (CFU) \\
\hline-0.08 (-0.24 to 0.07$), 0.309$ & $0.28 \pm 0.22$ & $0.37 \pm 0.27$ & Bacteroid (CFU) \\
\hline-0.08 (-0.13 to 0.30$), 0.777$ & $0.32 \pm 0.31$ & $0.23 \pm 0.30$ & Firmicutes(CFU) \\
\hline
\end{tabular}

FBS: Fasting blood glucose, BDI: Beck Depression Inventory, HDL: high density lipoprotein; LDL: low density lipoprotein, TG: Triglyceride, TC: Total cholesterol, SBP: Systolic blood pressure, DBP: diastolic blood pressure, MMP-9: Matrix metallopeptidase 9, hs-CRP: high sensitivity C-reactive protein, MDA: malondialdehyde, TAC: total antioxidant capacity. For other abbreviations see table 4 
Values are expressed as mean (SD)

a- Independent samples t-test

\section{Figures}

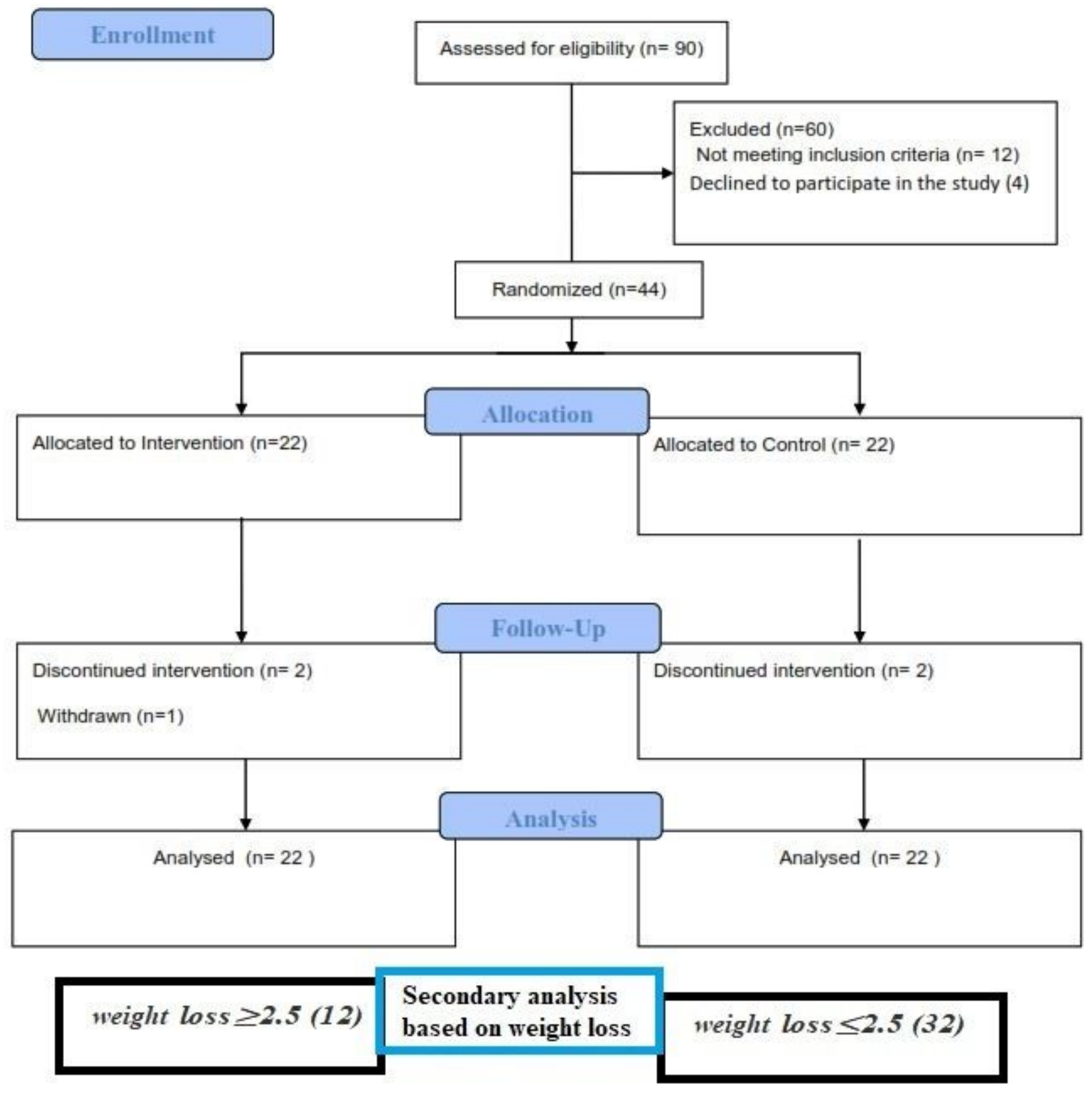

Figure 1 
CONSORT Flow diagram

Page 19/19 